\title{
Experimental Study on the Performance of Wind Stirring System with Different Working Fluids
}

\author{
Kang $\mathrm{YANG}^{1,}$, a , Xian-Peng SUN ${ }^{1, b^{,},}$, Yu GUO ${ }^{1}$, Sheng-Shan $\mathrm{BI}^{2}$ \\ and Xin-Yang QIU ${ }^{3}$ \\ ${ }^{1}$ College of Horticulture, Northwest A\&F University, Yangling, Shaanxi, China \\ ${ }^{2}$ Key Laboratory of Thermo-Fluid Science and Engineering, Ministry of Education, Xi'an Jiaotong \\ University, Xi'an 710049, China \\ ${ }^{3}$ College of Mechanical and Electric Engineering, Northwest A\&F University, Yangling, Shaanxi, \\ China \\ a1013629222@qq.com, 'sunxianpeng@nwsuaf.edu.cn
}

Keywords: Stirring and heating, Heating efficiency, Working fluid.

\begin{abstract}
In this work, a stirring and heating test system was built. An experiment at different rotational speeds within $1 \mathrm{~h}$ has been done for three working fluids: water, 46\# hydraulic oil and saturated $\mathrm{NaCl}$. Under the ideal state without considering the energy loss, the results show that the temperature rise of the working medium is faster as the rotation speed increases, the higher the heating efficiency. For example, the $46 \#$ hydraulic oil with smaller specific heat capacity is stirred at a speed of $500 \mathrm{r} / \mathrm{min}$ for $1 \mathrm{~h}$, and the temperature is increased by $27.5^{\circ} \mathrm{C}$. In the same rotational speeds, the change of temperature and heating efficiency of different working fluids is complicated, and the physical parameters, such as the mass density, the specific heat capacity and the kinematic viscosity, have a great influence on the heating efficiency.
\end{abstract}

\section{Introduction}

Wind-heating is a high-efficiency wind energy utilization technology that has emerged in recent decades. Wind-heating devices use wind energy to heat water and other working fluids, and provide heating for livestock houses, greenhouses, houses, bathrooms, etc. Reduce the demand for fossil energy such as coal and natural gas, and alleviate the increasingly serious environmental energy problems in the world [1]. At present, research on wind energy at home and abroad mainly focuses on wind power generation. In fact, the energy utilization rate of wind-heating is higher, the quality of wind is lower, the adaptability to wind conditions is stronger, and the energy storage problem is also easy to solve [2]. Wind energy is a clean renewable energy source with abundant reserves. The use of wind energy resources to convert it into heat for indoor heating in winter will greatly reduce heating costs. Therefore, research, development and application of wind-heating have obvious practical significance.

Wind-heating method mainly include stirring liquid heating, oil pressure damping hole heating, solid friction heating, hydraulic coupling type heating, eddy current heating, electric heating, compressed air heating and heat pump, etc. The heat-transfer mechanical energy is converted into heat energy with high efficiency, and the stirring paddle is easier to match with the wind turbine. There is no need to design an over-speed protection device. The heating device has a simple structure, reliable operation and low price [3]. Wind energy heating is the conversion of wind energy into mechanical energy, which is converted into heat energy, and the heat obtained is supplied to the heat dissipation system [4]. Specifically, the wind-heating uses wind energy as the power source, and the upper blade of the wind turbine rotates with the wind, and the main shaft of the heating device is rotated by the long-axis transmission. The main shaft of the heater is equipped with a stirring blade (rotor), and there is also a plurality of spoilers (stator) on the inner wall of the barrel. When the heating medium is injected into the heating barrel, the working fluid will undergo vigorous turbulent movement with the stirring blade, the flowing heating medium and the agitator blade, the spoiler, the working medium molecules collide with each other, friction, generate heat, 
and raise the temperature of the heating medium [5].

At present, there are few studies on wind-heating at home and abroad, and some researchers have carried out theoretical analysis on the combined heat of wind and solar energy, but there is no any related experiment [6, 7]. Agitating wind-heating device developed by Komatsu Manufacturing Co., Ltd., with a heating power of $25 \mathrm{~kW}, 30 \mathrm{~L}$ of water can be heated by a heater once to 15 to $20^{\circ} \mathrm{C}$ [8]. Agitating wind-heater manufactured by Lutoka, the Netherlands The designed power output thermal energy is $55 \mathrm{MJ} / \mathrm{h}$, the highest water temperature is $100{ }^{\circ} \mathrm{C}$, and the oil temperature is $150{ }^{\circ} \mathrm{C}$ [9]. Zhao Jianzhu of China Agricultural University experimented with wind-heating and heating, and studied the mixing blades and the spoiler. Based on the influence of size, shape and quantity on the absorbed power, the experimental data obtained by statistical methods are used to summarize the power absorption equation [10]. Shanghai maritime university $\mathrm{Hu}$ Yihuai put forward a kind of stirring and the way of wind turbine heating by the joint action of damping, and set up a set of power of $5.5 \mathrm{~kW}$ test device, under the condition of environment temperature is $17{ }^{\circ} \mathrm{C}$ run $30 \mathrm{~min}$, the $32 \mathrm{~kg}$ anti-wear hydraulic oil from $12{ }^{\circ} \mathrm{C}$ to $108{ }^{\circ} \mathrm{C}$ [11]. Guiyan of Shanghai Electric Power College studied the heating effect of flat blades and cylindrical blades as the heating device for stirring the heating blades. The comparative analysis showed that the effect of the straight blade heating flow is ideal [12].

In this paper, a set of indoor stirring and heating test system was built, and the experimental study on stirring heat of three kinds of working fluids water, 46\# hydraulic oil and saturated $\mathrm{NaCl}$ solution, was carried out.

\section{Test Equipment and Methods}

The test bench consists of variable frequency motor, torque speed sensor, reducer, multi-channel temperature collector, torque meter, heat exchanger, frequency converter, etc. The experimental system is shown in Fig 1. Because the laboratory can't capture wind energy, the variable frequency motor replaces the wind energy as the power source, and the inverter output frequency is controlled by the frequency converter to simulate the wind speed. The design of the heater structure has a large effect on the heating efficiency. The size of the heater, the shape of the agitating blades, the number of blades, the number and size of the baffles, etc. all affect the flow dynamics of the working fluid in the heater. The agitator barrel designed for this test has an inner diameter of $425 \mathrm{~mm}$ and a barrel height of $450 \mathrm{~mm}$; the agitator rotor has a diameter of $280 \mathrm{~mm}$ and the number of blades is 6 ; the width of the baffle is $50 \mathrm{~mm}$ and the length is $350 \mathrm{~mm}$. Piece; the rotor is a disc straight type. The heater model is shown in Fig2. The outside of the heater is covered with insulation material to prevent heat loss; the inside of the heater A plurality of temperature probes are distributed on the outer barrel wall and the room temperature, and the temperature data is recorded by the temperature collector; the torque meter records the torque and power data. The whole test platform is shown in Fig 3. The main equipment for the test is shown in Table 1.

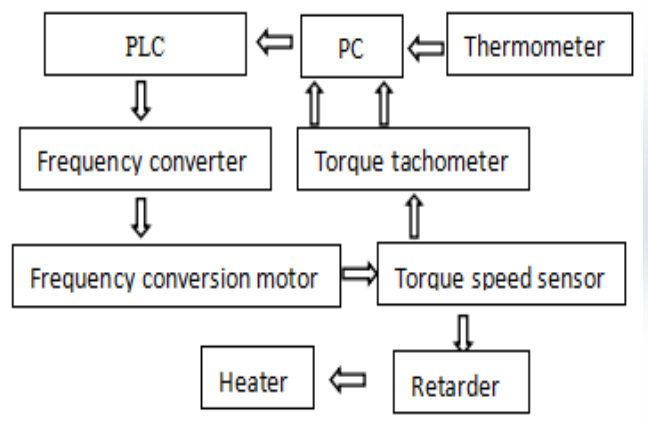

Fig. 1 Diagram of experiment system

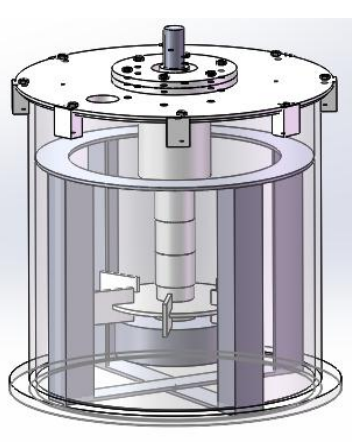

Fig. 2 Heater model

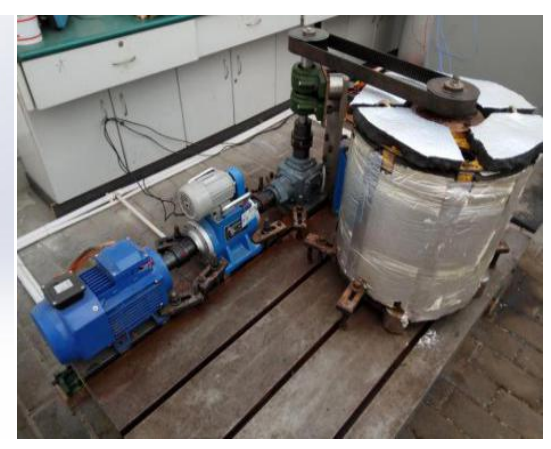

Fig. 3 experimental platform 
Table 1 Main equipment for heat stirring system

\begin{tabular}{ccc}
\hline Device name & Number & Model and main performance parameters \\
\hline Electric machinery & 1 & YVP100L-4 50 Hz 2.2 kW \\
Frequency converter & 1 & VFD037B43A \\
Torque speed sensor & 1 & $\mathrm{JC1A} \quad 0-50 \mathrm{~N} \cdot \mathrm{m} \quad \pm 0.2 \% \mathrm{~F} \cdot \mathrm{S}$ \\
Retarder & 1 & T6-4 $1-1-\mathrm{U}-\mathrm{B} 3 \quad 4: 1$ \\
Temperature collector & 1 & AT4508Multichannel temperature tester \\
Torque meter & 1 & $\mathrm{JW}-3 \quad$ RS 232 \\
\hline
\end{tabular}

\section{Test Methods}

During the experiment, the heat absorbed by the heating medium is [1]:

$$
\mathrm{Q}=\Delta \mathrm{tmc}
$$

In the formula: $\Delta \mathrm{t}$ is the temperature difference, ${ }^{\circ} \mathrm{C} ; \mathrm{m}$ is the quality of the working medium, $\mathrm{kg}$; $\mathrm{C}$ is the specific heat capacity of the working fluid, $\mathrm{J} /\left(\mathrm{kg} \cdot{ }^{\circ} \mathrm{C}\right)$.

The input power is:

$$
\mathrm{P}=\mathrm{P}^{\prime} \eta_{1}{ }^{2} \eta_{2} \eta_{3} \eta_{4}
$$

In the formula: $\mathrm{P}^{\prime}$ is Average power, $\mathrm{W} ; \eta 1$ is the coupling transmission efficiency, $0.99 ; \eta_{2}$ is the rolling bearing transmission efficiency, $0.99 ; \eta_{3}$ is belt drive transmission efficiency, $0.98 ; \eta_{4}$ is the transmission efficiency of the reducer, 0.96 .

Heating efficiency [1]:

$$
\eta=\mathrm{Q} / \mathrm{Pt}{ }^{*} 100 \%
$$

In the formula: $\mathrm{Q}$ is the working medium to absorb heat, $\mathrm{J} ; \mathrm{P}$ is the input power, $\mathrm{W} ; \mathrm{t}$ is the data measurement time, $\mathrm{s}$.

\section{Test Process}

This test uses water, $46 \#$ hydraulic oil and saturated $\mathrm{NaCl}$ solution, of which water and oil are the most common liquid working fluid [9]. The basic parameters of the three working fluids are shown in Table 2. The speed of the motor controlled by the frequency converter is $300 \mathrm{r} / \mathrm{min}, 400 \mathrm{r} / \mathrm{min}$, $500 \mathrm{r} / \mathrm{min}$; four T-type thermocouples are distributed inside and outside the heater, and the temperature is recorded every minute throughout the experiment; the torque meter records the torque, power and speed data every minute; the working volume is $20 \mathrm{~L}$; the entire experiment lasts for $1 \mathrm{~h}$.

Table 2 Basic parameters of three working fluids

\begin{tabular}{cccc}
\hline Working fluids & Density & Specific heat capacity & Boiling point \\
\hline water & $1000 \mathrm{~kg} / \mathrm{m} 3$ & $4.2 \mathrm{~J} /\left(\mathrm{kg} \cdot{ }^{\circ} \mathrm{C}\right)$ & $100{ }^{\circ} \mathrm{C}$ \\
$46 \#$ hydraulic oil & $865 \mathrm{~kg} / \mathrm{m} 3$ & $1.884 \mathrm{~J} /\left(\mathrm{kg} \cdot{ }^{\circ} \mathrm{C}\right)$ & $290{ }^{\circ} \mathrm{C}$ \\
saturated $\mathrm{NaCl}$ solution & $1330 \mathrm{~kg} / \mathrm{m} 3$ & $3.2 \mathrm{~J} /\left(\mathrm{kg} \cdot{ }^{\circ} \mathrm{C}\right)$ & $110^{\circ} \mathrm{C}$ \\
\hline
\end{tabular}




\section{Test Results and Analysis}

\section{Analysis of the Heating Characteristics of the Same Working Fluid at Different Speeds}

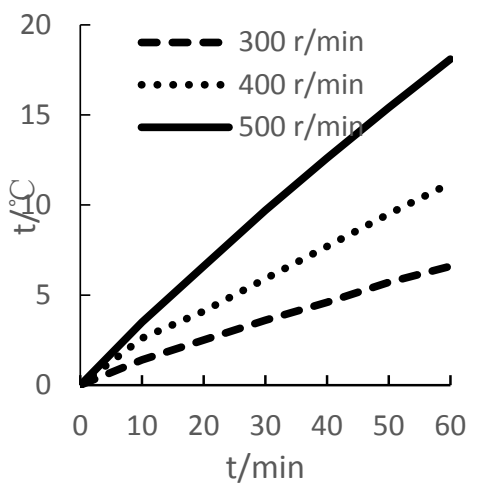

a

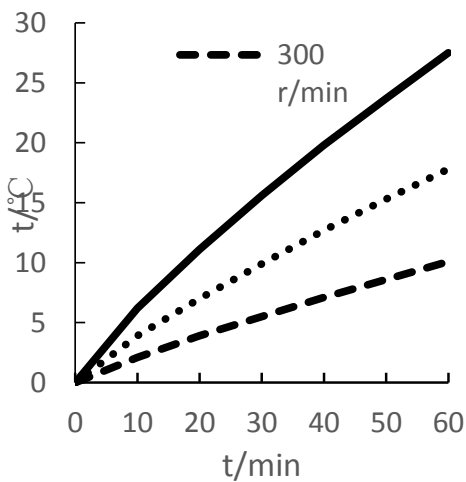

b

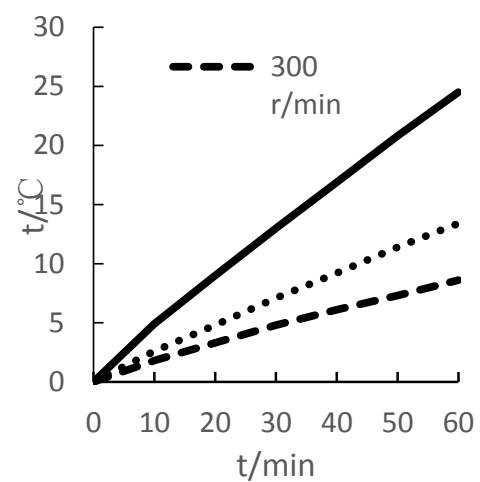

$\mathrm{c}$

Fig. 4 a, b, c are the temperature rise curve at different speeds when the working fluid is water, 46\# hydraulic oil, saturated $\mathrm{NaCl}$ solution.

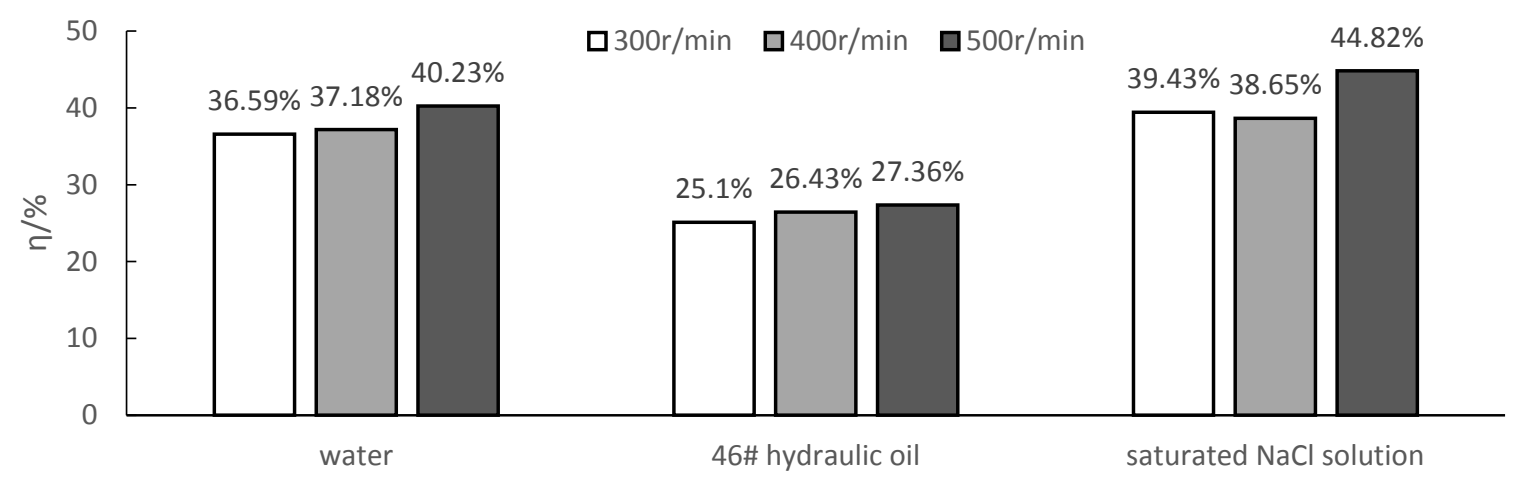

Fig. 5 Comparison of thermal efficiency of three working fluids of water, 46\# hydraulic oil and saturated $\mathrm{NaCl}$ solution at no speed.

It can be seen from Fig. 4 that under the same stirring time, as the rotational speed increases, the temperature rise of the same working medium increases, that is, as the stirring time is longer, the working temperature is higher, and as the rotational speed increases, the slope of the temperature change curve increases accordingly. When the speed is $300 \mathrm{r} / \mathrm{min}$, the water temperature rises by $6.6{ }^{\circ} \mathrm{C}$ in $1 \mathrm{~h}$, the temperature of $46 \#$ hydraulic oil increases by $10.1{ }^{\circ} \mathrm{C}$, the temperature of saturated $\mathrm{NaCl}$ solution increases by $8.6^{\circ} \mathrm{C}$; when the speed is $500 \mathrm{r} / \mathrm{min}$, the water temperature increased by $18.1^{\circ} \mathrm{C}$ within $1 \mathrm{~h}$, the temperature of $46 \#$ hydraulic oil increased by $27.5^{\circ} \mathrm{C}$, and the temperature of saturated $\mathrm{NaCl}$ solution increased by $24.5^{\circ} \mathrm{C}$. It can be seen from Fig. 5 that in the same stirring time, with the increase of the rotational speed, the heating efficiency of the same working fluid increases slowly, the rotational speed increases from $300 \mathrm{r} / \mathrm{min}$ to $500 \mathrm{r} / \mathrm{min}$, and the heating efficiency of water in $1 \mathrm{~h}$ from $36.59 \%$ rose to $40.23 \%$, the heating efficiency of $46 \#$ hydraulic oil increased from $25.1 \%$ to $27.36 \%$, and the heating efficiency of saturated $\mathrm{NaCl}$ solution increased from $39.43 \%$ to $44.82 \%$, of which $46 \#$ hydraulic oil has the lowest heating efficiency, far lower than water and the heating efficiency of saturated $\mathrm{NaCl}$ solution, the heating efficiency of saturated $\mathrm{NaCl}$ solution is slightly higher than the heating efficiency of water. 


\section{Analysis of the Heating Characteristics of Different Working Fluids at the Same Speed}

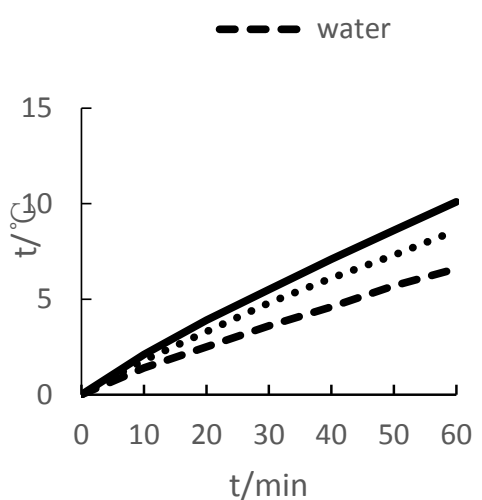

$\mathrm{a}$

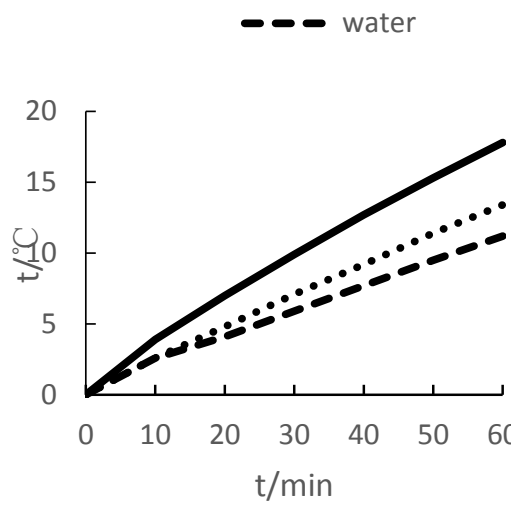

b

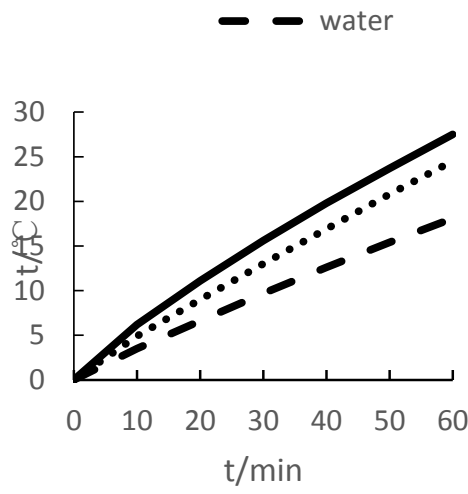

Fig. 6 a, b and c are the temperature rise curves of different working fluids at $300 \mathrm{r} / \mathrm{min}, 400 \mathrm{r} / \mathrm{min}$ and $500 \mathrm{r} / \mathrm{min}$ respectively.

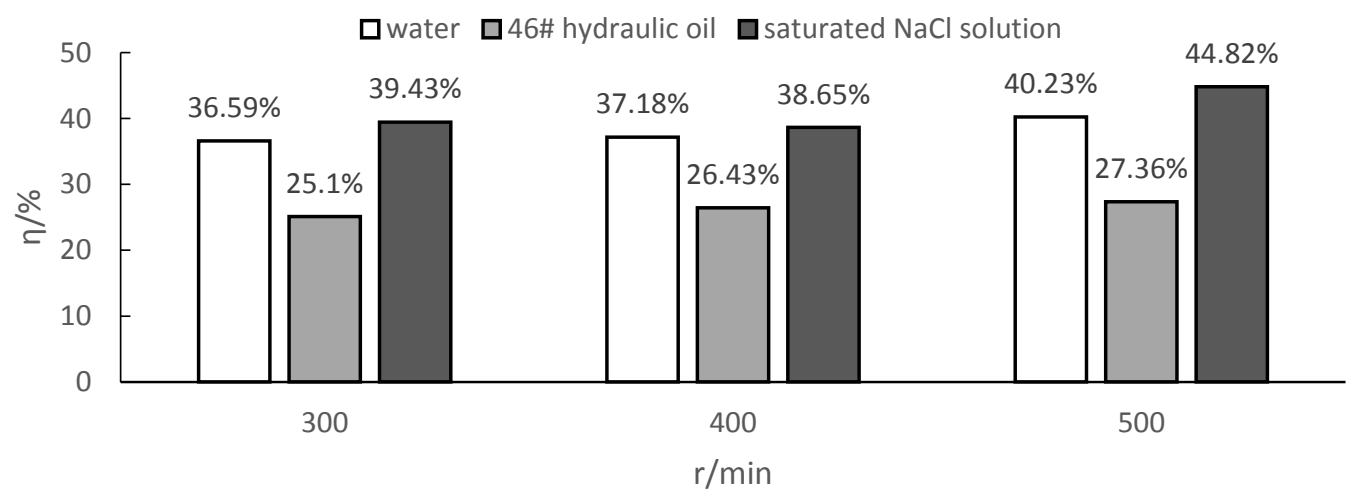

Fig. 7 Comparison of the heating efficiency of different working fluids at $300 \mathrm{r} / \mathrm{min}, 400 \mathrm{r} / \mathrm{min}$ and $500 \mathrm{r} / \mathrm{min}$

It can be seen from Fig. 6 that under the same same speed condition, the temperature rise of $46 \#$ hydraulic oil is the fastest, the temperature rise of water is the slowest, and with the increase of the rotational speed, the temperature rise of the hydraulic oil is still the highest. When the speed is 300 $\mathrm{r} / \mathrm{min}$, the temperature rise of $46 \#$ hydraulic oil is $3.5^{\circ} \mathrm{C}$ higher than that of water. When the speed is $500 \mathrm{r} / \mathrm{min}$, the temperature rise of $46 \#$ hydraulic oil is $9.4^{\circ} \mathrm{C}$ higher than the water temperature, that is, with the increase of speed, 46 \#the change in temperature rise of hydraulic oil is more obvious. It can be seen from Fig. 7 that the saturated $\mathrm{NaCl}$ solution has the highest heating efficiency under each speed condition, and the 46\# hydraulic oil has the lowest heating efficiency. At $500 \mathrm{r} / \mathrm{min}$, the heating efficiency of the saturated $\mathrm{NaCl}$ solution is $17.46 \%$ higher than that of the 46\# hydraulic oil.

\section{Conclusions}

In this paper, a set of indoor stirring and heating test system was built, and the experimental study on stirring heat of three kinds of working fluids water, 46\# hydraulic oil and saturated $\mathrm{NaCl}$ solution, was carried out. The main results are as follows:

(1) It is feasible to use stirred liquid heating mode, and the heat effect is remarkable. With the increase of rotating speed, the temperature rise and heat efficiency of the same working substance will increase and change.

(2) The comparative analysis of the three kinds of stirring heating working fluids shows that the 
46\# hydraulic oil with smaller specific heat capacity can obtain higher heating temperature. When the speed is $500 \mathrm{r} / \mathrm{min}$, the temperature is increased by $27.5^{\circ} \mathrm{C}$ after stirring for 1 hour. As the agitation time increases and the agitation speed increases, the temperature will continue to rise.

(3) At the same speed, the change of temperature and heating efficiency of different working fluids is more complex, and the physical parameters such as density, specific heat capacity and kinematic viscosity of the working material also have a great influence on the heating efficiency.

\section{Acknowledgement}

This research was financially supported by the Science and Technology Planning Project of Shaanxi Province, China (2017NY-154 and 20116KTCL02-02), and Major project of key research and development program in Ningxia Autonomous Region, China (20170919000001).

\section{References}

[1] Su Wenjuan, Li Yongguang. Experimental Research of Wind Stir Heating [J]. Journal of Shanghai University of Electric Power, 2016(03):274-276.

[2] Li Huashan, Feng Xiaodong, Liu Tong. Research progress of wind induced heat technology in China [J]. Solar Energy, 2008(09):37-40.

[3] Wang Shirong, Shen Dechang, Liu Guoxi. Wind induced water and wind induced heat [M]. Beijing: Science Press. 2012168-175.

[4] Liu Yang,Hu Yihuai.Parameter design of stirred wind heating device[J].Acta Energiae Solaris Sinica,2014, 35(10):1977-1980.

[5] Wu Shuyuan, Fan Chuiwen. Wind induced heat device [J]. Renewable Energy Resources, 1992(1):16-18.

[6] Elkinton M R, Mcgowan J G, Manwell J F. Wind power systems for zero net energy housing in the United States [J]. Renewable Energy, 2009, 34(5):1270-1278.

[7] Sateikis I, Lynikiene S, Kavolelis B. Analysis of feasibility on heating single family houses in rural areas by using sun and wind energy [J]. Energy \& Buildings, 2006, 38(6):695-700.

[8] Wang Yuankai. Current situation of wind induced heat in foreign countries [J]. Solar Energy, 1987(3):13-15.

[9] Song Jianfeng. Direct conversion of wind energy to heat energy [J]. Cereals and Oils Processing, 1988(1):39-41.

[10] Zhao Jianzhu. Research and experiment on wind induced heat [D]. China Agricultural University, 2006.

[11] Hu Yihuai, Han Zhongjian, Zhang Huawu. Experimental study on the stirred \& damped wind-heating system [J]. Energy Engineering, 2012(5):22-24.

[12] Gui Ting, Li Yongguang, Zhang Lihua. Experimental Study of Heating by Direct Stirring with Flat Blades and Cylindrical Blades [J]. Journal of Shanghai University of Electric Power, 2015, 31(2): 156-160. 